\title{
Montenegro als exotischer Raum in deutschsprachigen Reiseberichten in der ersten Hälfte des 19. Jahrhunderts
}

\author{
Schlüsselwörter: Reiseberichte, Montenegro, Njegoš Zeiten, Raumdarstellung, \\ exotisch, Exotismus
}

DOI: $10.4312 /$ ars.13.2.147-162

\section{Einleitung}

Der vorliegende Aufsatz befasst sich mit der Perzeption des Raumes in deutschsprachigen Reiseberichten über Montenegro aus der ersten Hälfte des 19. Jahrhunderts, als das "Land der Schwarzen Berge" ${ }^{1}$ mit seinem allseits bekannten Fürstbischof, Vladika Petar II. Petrović Njegoš (1813-1851) die Neugier europäischer Reisender weckte. ${ }^{2}$ In Anbetracht der Tatsache, dass sowohl rein geographische als auch subjektive, aus persönlichen Wahrnehmungen entstandene Darstellungen des Raumes ein unausweichlicher, integraler Bestandteil von Reiseberichten sind (Gazda, Tinecka, 2016, 851), werden im vorliegenden Artikel die Darstellungen des Raumes in deutschen Reiseberichten, die aus der Zeit stammen, als der Fürstbischof Njegoš das Land regierte (1830-1851), identifiziert, und unter den unterschiedlichen Aspekten des Exotismus im Zusammenhang mit der eurozentrisch geprägten Perspektive der dem westlichen Kulturkreis angehörenden Reisenden/Autoren betrachtet und analysiert.

1 In der zweiten Hälfte des 15. Jahrhunderts wurde die Bezeichnung Zeta eine Zeitlang durch den volkstümlichen Namen Crna Gora ersetzt, was wörtlich übersetzt Schwarze Berge bedeutet. Crna Gora (in der slawischen-kyrilischen Form) wurde erstmal 1296, in einer Charta des serbischen Königs Milutin erwähnt. (Miljić, 2016, 386-387) Laut der Enzyklopädie Jugoslawiens (1982) soll Crna Gora 1348 zum ersten Mal in italienischen Quellen als Černa Gora erwähnt und als Montagna Negra, Montenegro oder Monte Negro bezeichnet worden sein. So fand die italienische Bezeichnung Montenegro Eingang in die westeuropäischen Sprachen.

2 Petar II. Petrović Njegoš war der jüngste Fürstbischof von Montenegro und einer der berühmtesten Dichter in der montenegrinischen Literaturgeschichte. Er strebte nicht nur nach der staatlichen Unabhängigkeit Montenegros vom Osmanischen Reich, sondern auch nach Modernisierung der montenegrinischen Gesellschaft und versuchte, die traditionellen kämpferischen Lebensmuster, wie die Blutrache, zugunsten von Gepflogenheiten, die er während seiner Reisen nach Italien, Österreich und Russland kennenlernte, abzubauen. In Cetinje, der damaligen Hauptstadt Montenegros, gründete er eine Druckerei und erste Schulen. Außerdem ist er Autor historischer und philosophischer Werke: Der Strahl des Mikrokosmos (Luča Mikrokozma, 1845), Der Bergkranz (Gorski vijenac, 1847), Der falsche Zar, Šćepan der Kleine (Lažni car, Šćepan Mali, 1851). 
Einzugehen war hierbei auf die Fragestellung, inwieweit sowohl dokumentarische als auch künstlerische Darstellungen des Raumes in frühen Reiseberichten über Montenegro zu dessen Bild als außereuropäisches, geradezu exotisches Land beigetragen haben. Da Reiseberichte über Montenegro nicht isoliert von Reiseberichten über den Balkan im Allgemeinen betrachtet werden können, ist dies ein Bild, das von Verallgemeinerungen und Reduktionismus geprägt ist (Krivokapić, 2017, 3-4). Dieses Bild reicht bis tief in das 20. Jahrhundert hinein, und dessen Implikationen und Modifikationen werden von Maria Todorova in Die Erfindung des Balkans dekonstruiert. ${ }^{3}$

Der Exotismus wird im vorliegenden Aufsatz in Zusammenhang mit den Theorien des Postkolonialismus und Orientalismus allgemein als "jede Form der Orientierung auf das Fremde” verstanden, "die inhaltlich durch ihren Gegenstandsbereich bestimmt wird" (Badenberg, 2007, 220). Er bezieht sich auf die Rezeption des Fremden bzw. Unbekannten in einer anderen und in der Regel "größeren Kultur". Das Fremde ist Teil einer zeitlich und/oder örtlich entfernten Kultur und wird sowohl positiv als auch negativ konnotiert: einerseits als ursprünglich, natürlich, authentisch, attraktiv, vielfältig, antiklassizistisch, andererseits unzivilisiert, barbarisch, ignorant, wild, gefährlich, unangenehm anders. Der positiv konnotierte Exotismus taucht in den Reiseberichten über Montenegro vor allem in ethnographischen Darstellungen und vielfach in Form von Veranschaulichungen der traditionellen Gastfreundschaft, Ehrenhaftigkeit oder einer starken Verbundenheit mit Traditionen insgesamt auf, während der negative in den legendären Beschreibungen von Kriegsbräuchen, wie beispielsweise dem Zur-Schau-Stellen abgehackter Köpfe getöteter Feinde, in Erscheinung tritt (Popović, 2015, 256).

In diesem Artikel soll jedoch gezeigt werden, dass Montenegro auch durch typisierte Darstellungen des Raumes als exotisches Land perzipiert wurde, auch wenn diese überwiegend als dokumentarisch und nicht künstlerisch zu kategorisieren sind. Allein in der Schilderung der Anreise in die damalige Hauptstadt Cetinje von der Stadt Kotor aus wird beispielsweise die mit Attributen wie "schroff", "steil", "einsam", "nackt" beschriebene Landschaft (Stieglitz, 1941, 28-29) eine Determinante, ja gar eine Art Rechtfertigung für das Raue und Wilde in der Natur der Montenegriner.

Wie für Reiseberichte allgemein charakteristisch, wird die objektive geographische Darstellung der Landschaft - hier mittels Attribuierung - schnell durch eine subjektive Verknüpfung angereichert: “jeder Steinblock eine Schanze, jede Felswand eine Festung" (Stieglitz, 1941, 24). Und gerade die Art und Weise der Subjektivierung der Außenwelt, die fast immer Teil einer anderen Kultur als die des Reisenden ist, ist in den Reiseberichten reizvoll (Gazda, Tinecka, 2016, 851). 
Wegen der Positionierung, die Reiseberichte zwischen den Kulturen einnehmen und wegen ihrer Eigenschaft als hybrides literarisches Genre, das sich am Rande der Literatur bzw. an der Grenze zwischen Literatur und Dokumentation bewegt, werden Reiseberichte in diesem Aufsatz als eine literarische Gattung verstanden, die die Fülle an Informationen und Kenntnissen über das unbekannte Land und dessen Leute und Kultur festhält und dadurch "dieser [fremden Kultur] gegenüber einstellungsprägend wirkt” (Schuster, 2007, 641).

In der Absicht, die materielle Welt, auf die der Reisende zum ersten Mal dergestalt trifft, glaubhaft und objektiv darzustellen, oftmals auch seine Leser von der Wahrhaftigkeit seiner Aussagen zu überzeugen suchend, betrachtet er das Unbekannte jedoch durch "sein eigenes ideologisches, kulturelles, intellektuelles und rhetorisches Prisma" (Popović, 2015, 7) und gleitet damit unweigerlich in die Subjektivität. Im Spannungsfeld zwischen der Absicht zur Objektivität und der ausdrücklich subjektiven Erzählerperspektive sowie der subjektiv getroffenen Auswahl der dargestellten Begebenheiten und Mittel der Darstellung wurden in den Reiseberichten über Montenegro im 19. Jahrhundert in Form des Exotismus prägende ethnobzw. eurozentrische und koloniale Stereotype vermittelt, jedoch auch Kulturkritik angedeutet. Dies wird am Beispiel des Exotismus bei den Darstellungen des Raumes in deutschsprachigen Reiseberichten gezeigt.

\section{Reiseliteratur über Montenegro}

Den ersten Reisebericht über Montenegro schrieb im Jahr 1614 Mariano Bolizza, ein Italiener aus Kotor. ${ }^{4}$ Danach erschienen bis nach der napoleonischen Eroberung Dalmatiens und der Bucht von Kotor nur wenige weitere Werke. ${ }^{5}$ Die einflussreichste

4 Marijan Bolica hat im Jahr 1614 auf Italienisch eine Reisebeschreibung mit dem Titel Relatione et descrittione del Sangiacato di Scutari verfasst. Darin lassen sich geographische und statistische Informationen sowie eingehende Beschreibungen politischer und ökonomischer Begebenheiten auf dem Territorium des Sandschaks Shkodra finden. Das Werk enthält auch wichtige Angaben über das archäologische und kulturelle Erbe, die Bevölkerung und deren Sitten.

5 Kotor war damals Teil der Republik Venedig. Nach dem Preßburger Frieden (Dezember 1805) musste Österreich ehemalige venezianische Besitztümer in Dalmatien und der Bucht von Kotor an Frankreich abtreten, was bedeutete, dass Napoleon in die montenegrinische Nachbarschaft kam. Russland war gegen das französische Eindringen in diese Gebiete, womit sich im RussischFranzösischen Krieg eine neue Front auftat, an der Montenegriner kämpften. Anfang 1806 besetzten Montenegriner zusammen mit russischen Truppen die Bucht von Kotor. Der MontenegrinischRussische Krieg gegen Frankreich wurde 1807 beendet. Mit dem Frieden von Tilsit zwischen Napoleon und dem russischen Zaren Alexander gelangten diese Gebiete wieder an Frankreich. Die französische Herrschaft in der Bucht von Kotor und dem Küstenland währte jedoch nur bis 1813 . Im Januar 1814 eroberte der montenegrinische Metropolit Petar I. Petrović Njegoš (1748-1830), der Onkel des berühmten Petar II. Petrović Njegoš, die Bucht von Kotor und das Küstenland. Zu diesem Zeitpunkt wusste er jedoch nicht, dass die anti-napoleonische Koalition die Illyrischen Provinzen und die Bucht von Kotor Österreich angeboten hatte, was mit dem Friedensvertrag von Paris im Mai 1814 auch bestätigt wurde. Der Vertrag zur Übergabe dieser Gebiete an Österreich 
Schrift aus dieser Epoche ist Historische und politische Reisen nach Montenegro (Voyages historiques et politiques au Monténégro, 1820) des französischen Obersten Vialla de Sommières, die "den Beginn einer breiteren Aufmerksamkeit für Montenegro in Europa" darstellt (Konstantinović, 1960, 82-83). Das eigentliche Interesse an diesem kleinen Land erweckte jedoch erst Njegoš dank seines Rufes in Europa, das Land reformieren und an Europa annähern zu wollen.

Die direkte Grenze mit dem Kaisertum Österreich trug dazu bei, dass die größte Anzahl der Reiseberichte des 19. Jahrhunderts aus der Feder von deutschsprachigen Reisenden stammt. Hinzu kommt, dass im Jahr 1837 im Verlag Cotta auf Deutsch das Werk Montenegro und die Montenegriner von Vuk Stefanović Karadžić (1787-1864) erschien, das einen außerordentlich großen Einfluss auf künftige Reiseberichte von Deutschsprachigen, die das kleine Land bereisten, hatte. ${ }^{6}$ Nur ein Jahr nach dem Erscheinen dieses Werkes, im Frühjahr 1838, besuchte König Friedrich August II. von Sachsen (1797-1854) Montenegro.7 Sein Begleiter, der italienische Botaniker, Bartolomeo Biasoletto, hielt die Reise des Königs in einem Werk mit dem Titel Relazione del viaggio fatto nella primavero dell' anno 1838 dalla Maesta del Re Frederico Auguste di Sassonia nell' Istria, Dalmazia e Montenegro fest. Die deutsche Übersetzung dieses Reiseberichtes aus dem Jahr 1842 (Reise Sr. Majestät des Königs Friedrich August von Sachsen durch Istrien, Dalmatien und Montenegro im Frühjahr 1838) ${ }^{8}$ trug ebenfalls dazu bei, dass weitere Wissbegierige aus dem deutschsprachigen Raum nach Montenegro reisten, sodass bis zum Ende des Ersten Weltkrieges mehr als 30 Reiseberichte und unzählige Zeitungsartikel von deutschsprachigen Schriftstellern, Journalisten, Botanikern, Geographen, Politikern usw. über das kämpferische montenegrinische Volk und sein Land im Dreieck zwischen slawischer, türkischer und westlicher Welt veröffentlicht wurden. Diese Werke und ihre Autoren ${ }^{9}$ haben die deutsch-montenegrinischen Beziehungen im frühen 20. Jahrhundert geprägt und

wurde von Montenegro im Juni 1814 unterschrieben. Die nächsten 100 Jahre sollte Montenegro nun die Habsburgermonarchie als Nachbarstaat haben. Die Grenze begann bei Kufin, ungefähr zehn Kilometer südlich des Kastells Lastva, und dehnte sich bis Dragalj in der Nähe des Dorfes Grahovo aus (Andrijašević, 2016, 134-137) - siehe Abbildung I.

6 Die serbische Originalfassung wurde erst unlängst entdeckt und veröffentlicht. Die deutsche Übersetzung dürfte auf W. Hoppe zurückgehen. Jernej Kopitar (1780-1844) schreibt nach dem Erscheinen dieses Buches an Karadžić: "Cotta verlangt alle Ex. der Montenegriner nach Hause, weil sie ihm dort ganz ausgegangen. Sie glücklicher Autor!”. Und an anderer Stelle: "Nur Sie allein tragen Schuld daran, dass alle Welt nach Montenegro fährt." (Konstantinović, 1960, 82-83).

7 Der Besuch von Friedrich August II. stellt den ersten Staatsbesuch eines europäischen Herrschers in Montenegro dar, obwohl er, ein passionierter Botaniker, primär aus Interesse an der Botanik dieser Gebiete Dalmatiens und Montenegros bereisen wollte.

8 Die Übersetzung ins Deutsche wurde von Eugen Freiherr von Gutschmied verfasst.

9 Um nur einige von vielen zu erwähnen: Welden, Michahelles, Leopold von Ranke, Franz Petter, Wilhelm Hoppe, Purstel und andere. 
nachhaltig beeinflusst. ${ }^{10}$ Das Fundament hierfür wurde indirekt jedoch bereits durch die Werke aus der ersten Hälfte des 19. Jahrhunderts gelegt.

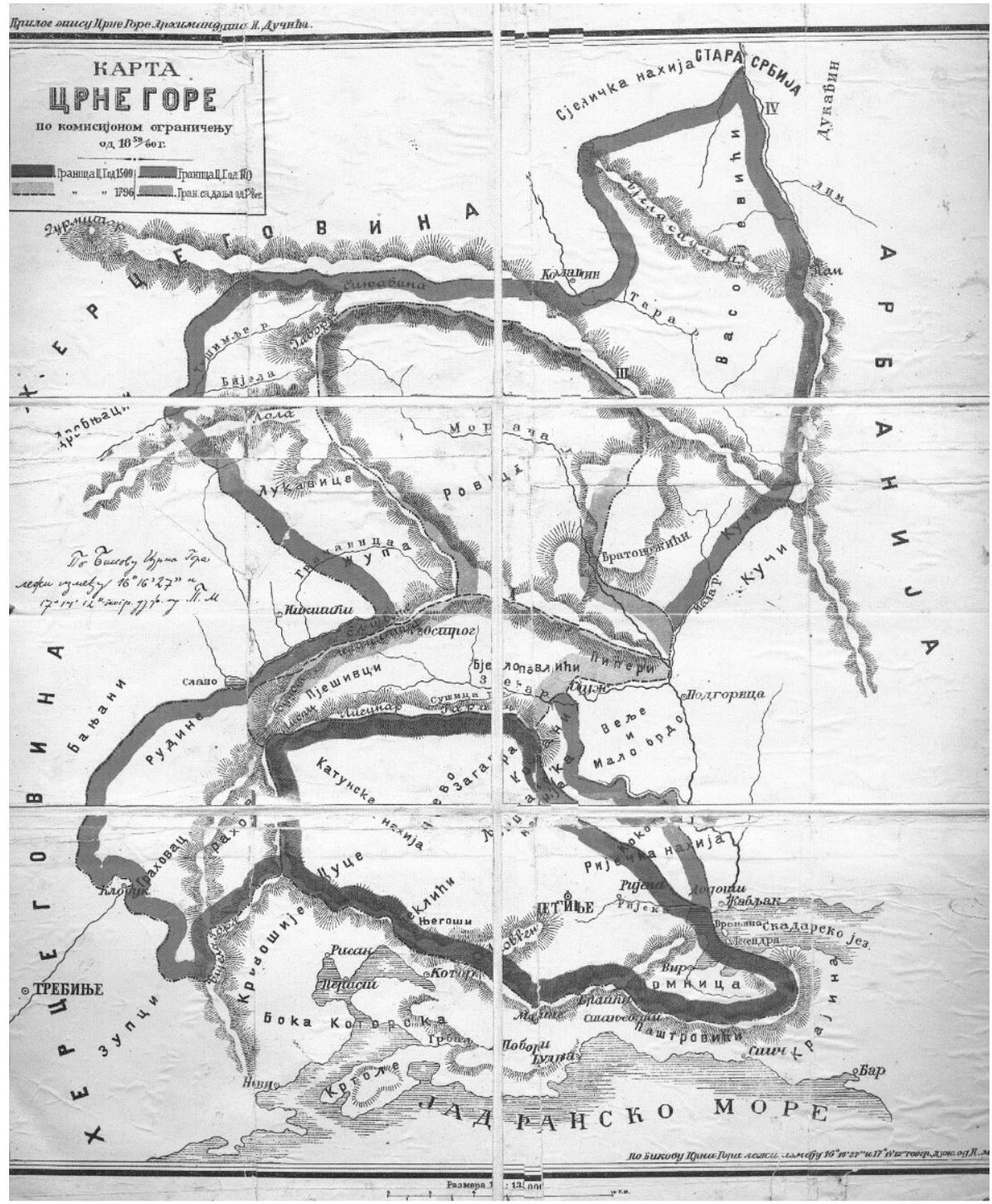

Abbildung 1: Quelle: Branko Pavićević, Istorija Crne Gore, Bd. 4, Sazdanje crnogorske nacionalne države 1796-1878, Podgorica 2004.

10 Vgl. Konstantinović, 1960; Krause, 2003; Medenica, 1927; Mojašević, 1955; Perišić, 1981. 


\section{Der Raum in Reiseberichten über Montenegro}

Für den vorliegenden Beitrag werden sechs Reiseberichte aus Njegoš Zeiten analysiert und interpretiert. Ausgeklammert wurden Werke von Autoren, die das Land nicht selbst bereist haben, auch wenn sie mitunter "äußerst eingehend und wahrheitsgetreu" sind (Konstantinović, 1960, 82). ${ }^{11}$

In den sechs analysierten Reiseberichten von Stieglitz (1841 und 1845), Ebel (1842-1844), Kohl (1851), Müller (1844) und Neigebaur (1851) wurden folgende Aspekte bei der Darstellung des Raumes einer näheren Betrachtung unterzogen: die Stadt Kotor als Gegensatz zur Stadt Cetinje, die steinige Landschaft, der gefährliche, steile Aufstieg von Kotor nach Cetinje, die Grenze Montenegros zum Kaisertum Österreich und zum Osmanischen Reich, das Kloster von Cetinje, private Häuser und Njegoš Residenz. ${ }^{12}$

Alle fünf Autoren erlebten Montenegro sowohl als geographischen als auch politischen Raum und konstruieren ihn in ihren Texten im Gegensatz zum damals österreichischen Kotor und Küstenland als eine radikale Fremde. Mit Ausnahme von Joseph Müller beginnen und enden alle Reisen in Kotor. Ihre Darstellungen der Stadt in der Bucht ähneln dem üblichen Bild Kotors als Grenzstadt, das in der damaligen wie heutigen Reiseliteratur über Montenegro vielfach zu finden ist Kotor als der letzte Ort, den die Zivilisation erreichte, ein reiches Handelszentrum, das Montenegro mit der entwickelten Welt verbindet (Popović, 2015, 259). Heinrich Stieglitz, dessen Buch Ein Besuch auf Montenegro mit einer umfangreichen Einleitung beginnt, in der detailliert diverse historische, geographische und demographische

11 So etwa die Skizze von Montenegro des Professors Franz Petter. Sie wurde im Jahr 1832 in der geographischen Zeitschrift Taschenbuch zur Verbreitung geographischer Kenntnisse veröffentlicht. Der Herausgeber dieser Zeitschrift, der Schriftsteller Johann Gottfried Sommer, der selbst ebenfalls niemals in Montenegro gewesen war, verfasste drei Jahre später, inspiriert durch Texte von Boue, Robert, Karadžić, v. Welden und Stieglitz, selbst einen Text mit dem Titel Zur Kenntnis von Montenegro. In diesem Zusammenhang sollte auch das Werk von Wilhelm Adolf Lindau nicht unerwähnt bleiben. Der Autor bearbeitete den Reisebericht des englischen Ägyptologen und Schriftstellers Sir John Gardner Wilkinson und veröffentlichte ihn 1849 unter dem Titel Dalmatien und Montenegro. Mit einem Ausfluge nach der Herzegowina und einer geschichtlichen Uebersicht der Schicksale Dalmatiens und Ragusa's in Leipzig.

12 Heinrich Wilhelm August Stieglitz (1801-1849) war Dichter und Autor etlicher Reiseberichte (Bilder des Orients, 1831). Er besuchte Dalmatien und Montenegro 1839. Wilhelm Ebel, Naturwissenschaftler, Botaniker und Privatdozent an der Universität zu Königsberg, bereiste Dalmatien 1841 und besuchte anschließend Kotor und Montenegro. Weitere persönliche Daten sind unbekannt. Johann Georg Kohl (1808-1878), Jurist und Stadtbibliothekar in Bremen, schrieb erfolgreiche Reisebeschreibungen über Russland und besuchte im Rahmen seiner Weltreisen Anfang der 1850er Jahre auch Montenegro. Joseph Müller (1811-1845) wurde als Arzt während der orientalischen Pest (1837/1838) als "Sanitätscommissär” nach Rumelien und Albanien gesandt und danach zum „Districtsphysiker zu Budva“ ernannt. Johann Ferdinand Neigebaur (1783-1866), ein preußischer Justizbeamter und Schriftsteller, verbrachte die Zeit seines Ruhestandes auf Reisen durch Europa und kam ebenfalls Anfang der 1850er Jahre nach Montenegro. 
Daten zu Montenegro angeführt werden, widmet sein erstes Kapitel der Stadt, die in den "aschgrauen Fels [Montenegros] hineingedrängt ist” (1841, 3). Für ihn ist Kotor der Ort, wo er zwischen den Stadtmauern und den Höhen "zum ersten male jene vielbesprochenen Bergsöhne" sah $(1841,5)$. Dem bunten, lebendigen Tableau des Basars in Kotor stehen als Kontrast "die grauen Felswände" gegenüber (1841, 10), von wo aus die Montenegriner vor nicht allzu langer Zeit wegen des Konflikts über den Verlauf der Grenze riesige Felsbrocken auf die österreichischen Truppen geworfen hatten. So wird Montenegro, das hinter den grauen Felswänden beginnt, gleich durch die räumliche Anordnung mit der drohenden Andersheit in Verbindung gesetzt, was danach noch mit einem Gleichnis erweitert wird:

Der Bewohner der Höhen steht $\mathrm{zu}$ dem in bürgerlichem Behagen eingerichteten Thalbewohner immer im Verhältnisse des Raubvogels zu der weidenden Heerde. [...] Wehe der friedlichen Heerde in den Thälern, wenn ein Sturm von Oben den Falten von der Höhe plötzlich allen mit einem Stoße ihre Rappen löste! (Stieglitz 1841, 28)

Dem ähnlich ist noch ein erweiterter Vergleich, in dem Johann Georg Kohl Montenegro mit einem Vulkan vergleicht, der "rings um sich her Ruinen geschaffen hat" (Kohl, 1851, 194). Das Bild entsteht jedoch in der Imagination des Reisenden aufgrund der Geschichten über die Zerstörung von Dubrovnik und dessen Umgebung durch montenegrinische Angriffe im Jahr 1806, während er mit eigenen Augen "diesen Vulkan, das himmelanstrebende tschernogorische Gebirge, recht freundlich und schön, von der Sonne anmuthig beschienen" (ebd., 194) sieht. Die Städtchen in der Bucht von Kotor beschreibt Kohl als malerisch, schön und zahm. Für das erste montenegrinische Dorf, Njeguši, benutzt er dann hingegen expressive räumliche Metaphern: "das höchste und unzugänglichste Felsennest" und "die wahre Akropolis von Montenegro" (ebd., 282), wobei beide Metaphern auf die fehlende Verbundenheit zwischen Montenegro und dem Europäischen verweisen.

Auch Johann Ferdinand Neigebaur setzt Montenegro anhand eines Vergleichs mit der "Höhe des Vesuvs", nur noch "steiler" und "gefährlicher" (Neigebaur, 1851, 61) in Verbindung. Die "Felsenschluchte, Felsengebirge und Felsenthäler", die "kein Ende" haben (ebd., 61), werden der zahmen Landschaft und dem "reizenden" Ufer der "herrlichen" Bucht (ebd., 30) entgegengesetzt. Oberhalb der Bucht von Kotor ist Montenegro als ein "Felsenlabyrinth", wo sich die Wohnungen von Menschen befinden, dargestellt, und das “wundert" den Autor (ebd., 57).

Paradoxerweise ist dieser Kontrast zwischen dem zivilisierten, österreichischen Kotor und dem wilden Land der Schwarzen Berge im zweiten Buch von Stieglitz am auffälligsten. Stieglitz kam fünf Jahre nach seiner ersten Reise zwar wieder in die Nähe 
des damaligen Montenegro, besuchte diesmal jedoch nicht dieses ,,in jeder Hinsicht so höchst merkwürdige[n] Stückchen Erde" (Stieglitz, 1845, 204). Er schreibt ausführlich über Kotor und seine "feine[n], parkartige[n] Umgebung" mit ihren "romantischen Inselchen" und der "ununterbrochene[n] Reihe freundliche[r] Ortschaften" (ebd., 209). Im starken Kontrast dazu steht "das starre Gränzgebirg von Montenegro" mit “seiner wilden Rauhheit” und seinen "grauen starren Felsenschanzen” (ebd., 209).

Nicht nur Kotor ist für Stieglitz ein Grenzort, den er als das Eigene erkennt, sondern auch diese kleinen freundlichen Ortschaften an der Küste, die als Tatorte montenegrinischer Angriffe "bedeutend gelitten haben" (ebd., 219). Auch in seinem ersten Reisebericht schenkt er der Grenze Montenegros sowohl zu Österreich als auch zum Osmanischen Reich viel Aufmerksamkeit. An dem Ort der unmittelbaren Berührung dreier "der Sitte und der Richtung nach so gänzlich verschiedlicher Grenzstaaten" (Stieglitz, 1841, 150) stehend, offenbart Stieglitz seine Begeisterung über die paradiesische Landschaft des fruchtbaren Tals Paštrovića, das mit seinen "sorglich bebauten Feldern" wesentlich positiver dargestellt wird als die grauen, nackten Spitzen Montenegros und die bewaldeten Höhen Albaniens. Er verbirgt nicht seine Freude über die Tatsache, dass gerade diese Landschaft "die südlichste Besitzung einer deutschen Macht” ist (ebd., 150). Ähnlich, wie er sich schon beim Schreiben über Kotor an den neuen Waffen erfreute, mit denen die österreichischen Truppen aus der Ferne "den Feind hinter seinem Felsenversteck aussuchen werden", tritt hier seine koloniale Perspektive in den Vordergrund, obwohl er selbst an mehreren Stellen den Montenegrinern Eroberungsbestrebungen vorwirft und damit seine eigenen kolonisatorischen Vorstellungen auf Njegoš und die Montenegriner projizierte. ${ }^{13} \mathrm{Da}$ er später noch die Ereignisse in der nahe der Grenze gelegenen Ortschaft Grahovo und direkt an der Grenze mit den Osmanen schildert, scheint es Stieglitz äußerst wichtig zu sein, dass auch diese Grenzlinie zu Montenegro aufrechterhalten wird. Dies insofern, als dass mit dieser räumlichen Begrenzung sichergestellt ist, dass die montenegrinische streitlustige Andersheit auf Distanz bleibt. Mit den Metaphern der "starken Schanze" und der "Bollwerke", die er für die Grenze benutzt (ebd., 151-152), unterstreicht er die Perspektive eines Übergeordneten, eines Beschützers der europäischen Werte vor dem drohenden Fremden, das er doch zwangsläufig als neue, unaufhaltsame Kraft erkennt. Umso mehr ist die Grenze bei ihm als Symbol der notwendigen Abgrenzung der Welten voneinander zu verstehen.

13 Es ist interessant, dass Stieglitz, wie in der Einleitung seines ersten Reiseberichtes über die Einwohnerzahl und Fläche des Landes zu erkennen ist, nicht nur die Messdaten aus objektiven Quellen übernimmt, sondern die Größe Montenegros auch mittels der Anzahl seiner Kämpfer beschreibt, die sich, wenn notwendig, aus unterschiedlichen Ecken des Landes innerhalb von 24 Stunden versammeln könnten, um anzugreifen oder einen Angriff zu erwidern (Stieglitz, 1841, XLIIXLIII). Damit wird die Raumvorstellung nochmals prägend mit dem Aspekt des Kämpferischen der Montenegriner verbunden. 
Die Grenze wird auch bei Neigebaur und Müller überwiegend in Zusammenhang mit den Festungen, Bastionen und Stadtmauern dargestellt. Neigebaur merkt an, dass in der Festung auf den "spitzen" Felsen oberhalb des Ufers die "Besatzung stets vor den Montenegrinern auf der Hut sein muß” (Neigebaur, 1851, 34). Die mit Schießscharten und Kanonen versehene Festung von Spuž, die von einem "beträchtlich tiefe[n] Graben" umringte Stadtmauer von Podgorica (Müller, 1844, 54-55) oder das "rund herum mit gemauerten Brustwehren versehen[e]" Kloster Stanjevići (ebd., 92) in Verbindung mit den ständigen Überfällen aus dem "rauhen" Hinterland (ebd., 54) implizieren die Nähe der Montenegriner auch als drohend. Die "berühmte Pastrovicer Ebene", die die natürliche Grenze zwischen Montenegro und dem österreichischen Dalmatien bildete, beschreibt Müller als “Erisapfel zwischen zwei Ländern” (ebd., 93), verabsäumt es jedoch nicht zu erwähnen, dass die Kulturen und Gebräuche der beiden Völker in viele Aspekten einander ähneln. Der Raum ist also als untrennbar verbunden mit den Menschen zu betrachten, die ihn besiedeln und beleben, wie Ebel an anderer Stelle andeutet (ebd., 40). Die Überschreitung der Grenze bezeichnet Ebel mit dem Begriff "Examen rigorosum" (ebd., 37) und bezieht sich damit auf die Begegnung mit einer Gruppe von Montenegrinern, die ihn und seine Begleitung als eine Art Kontrolle im nahe der Grenze einkreiste. Noch vor dieser Inspektion war die Tauglichkeit des Reisenden durch den "immer beschwerlichen Weg [...] von Felsblock zu Felsblock" bei der drückenden Hitze auf die Probe gestellt worden (ebd., 33).

Der Blick auf Land und Leute ist für alle Reisenden/Autoren somit mit einem gewissen Gefühl des Argwohns verbunden. Schon während des Aufstiegs von Kotor nach Cetinje wurde ihnen bewusst, dass sie einer anderen Welt begegnen würden, deren Bewohner in einer "fast ununterbrochene[n] Felsenwüstenei" (Kohl, 1851, 288) um ihr nacktes Überleben kämpfen müssen. Deshalb merkt Kohl an, dass der räumlich geprägte Name des Landes vielleicht überhaupt nichts mit der schwarzen Farbe der Berge zu tun haben könnte, sondern in Wahrheit vielleicht auf die "verwünschte, trübselige und schwarzmelancholische Rauhigkeit dieser Berge" verweisen könnte (ebd., 334).

Alle steinigen Wege führen nach Cetinje, und das Erste, das alle Reisenden noch aus großer Entfernung erblicken, ist ein viereckiger alter Turm, "dessen Zinnen die Montenegriner mit den Köpfen ihrer getöteten Feinde auszuschmücken pflegten" (Kohl, 1851, 295). Einige Jahre zuvor hatten Stieglitz, Ebel und Neigebaur ihn noch "mit neuem Schmuck reichlich versehen" (Stieglitz, 1841, 110), mit aufgestellten “Türkenköpfe[n] als Trophäen” (Neigebaur, 1851, 59) betrachten und sowohl als Symbol der schaurigen montenegrinischen Lebensrealität als auch als eindeutiges Zeichen einer bedrohlichen Exotik perzipieren können. In diesem Zusammenhang zweifelt Stieglitz an der Aufrichtigkeit der Modernisierungsbestrebungen Njegoš, 
solange sich direkt über seiner Residenz noch immer ein "Schädelturm" befindet (Stieglitz, 1841, 110).

Ebel bezeichnet Cetinje als "ein offenes Heerlager [...], ein Versammlungsort von Kriegern und Feldherrn” (Ebel, 1842-1844, 52), während Stieglitz die Stadt in einem seltsamen Kontrast, den zwei Hauptgebäude bilden, darstellt: die neu erbaute Residenz und das Kloster, das er als ein natürliches Bollwerk ansieht. Der Kontrast spiegelt die Dualität der weltlichen und kirchlichen Herrschaft wider bzw. den Kampf mit dem Schwert in der einen und dem Kreuz in der anderen Hand, was er später auch thematisiert; jetzt jedoch, solange es nicht mehr um den Kampf gegen Österreich, sondern gegen die Osmanen geht, betont er ihn in einem fast heroisierenden Ton. Dem entspricht auch die Metapher der Mauer "aus den Leibern seiner Söhne" (Stieglitz, 1841, 110), die Montenegro dauerhaft schützt.

In den mehr oder weniger detaillierten Beschreibungen der Residenz Njegoš und des Klosters ist sowohl bei Stieglitz als auch bei Ebel und Kohl das Interesse an den Räumen mit den Waffen auffällig. Diese Räume voll Waffen werden auch als Symbol der eigenartigen, kämpferischen Mentalität dieses merkwürdigen Volkes dargestellt.

Die Druckerei und die Schule werden vor allem im Kontext der Modernisierung als kulturelle Institutionen beschrieben, die Stieglitz mit der Möglichkeit eines Kampfes der anderen Art Montenegros gegen den Westen in Verbindung setzt, nämlich nicht mehr mit Waffen, sondern mit der Feder. In Zusammenhang mit der Bibliothek von Njegoš beschreibt Kohl auch nicht den Raum selbst, sondern thematisiert die europäische Ausbildung des Vladika sowie seine literarische Tätigkeit. In der Darstellung der Residenz mit den einfachen Zimmern Njegoš ist auch das BillardZimmer unvermeidlich, das bei Kohl z. B. etwas von dem Ambiente der europäischen Höfe überträgt. ${ }^{14}$

Als Gegensatz zur Residenz als halb-öffentlichem Raum stehen einerseits die einfache Gaststätte in Cetinje als öffentlicher Raum und andererseits die bescheidenen Wohnhäuser als privater Raum. Alle Räume verbindet die Metapher der offenen Tür - sei es, dass sie durch die Darstellung der Gastfreundschaft des Vladikas und der Bereitwilligkeit der wohlwollenden Montenegriner, in ihrer Armut Gäste gebührend zu empfangen, realisiert wird, oder wie bei Ebels durch die Beschreibung der einfachen Gaststätte, wo die Tür zu seinem Zimmer nicht versperrt werden konnte, sodass jeder

14 Untrennbar verbunden mit der Darstellung der Residenz ist das Porträt des Vladika selbst, der mit seiner imposanten Erscheinung alle anderen Aspekte der Beschreibung der montenegrinischen Hauptstadt in den Schatten stellt, was jedoch nicht direkt zum Thema dieses Aufsatzes gehört. Alle in direktem Kontakt entstandenen Porträts sind positiv konnotiert, jedoch verurteilt Stieglitz in seinen beiden Reiseberichten die angeblichen Eroberungsbestrebungen des Vladikas und bezeichnet dessen Herrschaft als "tyrannisch" (Stieglitz, 1841, 44). 
das Zimmer einfach betrat, um den Ausländer zu sehen (Ebel, 1842-1844, 42-43). Dadurch wird nicht nur symbolisch klar, sondern auch literarisch gelungen dargestellt, wie die voneinander abgegrenzten Welten aufeinander treffen. Die subjektive, eurozentrische Perspektive des Autors tritt wiederum in den Vordergrund, wenn das Fehlen von "Öfen, Abtritte[n] und Senkgruben" (Müller, 1844, 99) in privaten Häusern geschildert wird, wodurch auf den niedrigen Zivilisationsgrad der Montenegriner hingewiesen wird. Ähnlich verhält es sich mit der Darstellung der Straßen - die neu gebauten Straßen weisen auf die gelungene Modernisierung und den Fortschritt hin, "die Beschaffenheit des Landes" hingegen darauf, dass sich die Geschichte Montenegros "nicht von fernen Jahrhunderten herleitet" (Neigebaur, 1851, 62).

$\mathrm{Zu}$ erwähnen ist des Weiteren noch eine geradezu paradiesische Darstellung des Landstrichs Crmnica bei Stieglitz. Der Blick auf ein Stück dieses fruchtbaren, bebauten montenegrinischen Landes zwischen dem Skadarsee und der Adriaküste erweckt in der Phantasie des Reisenden, der bisher Montenegro ausschließlich als Felswüste kannte, das Bild Arkadiens, und "ein lichter Traum völkerbeglückender Friedensherrschaft zog durch [s] eine Seele" (Stieglitz, 1841, 142). Diese paradiesische Darstellung deutet die Alternative eines ruhigen Lebens der Montenegriner ohne Krieg und Eroberungen bzw. die Möglichkeit einer Zivilisierung an, die auch wirtschaftlichen und kulturellen Fortschritt bringen und die allgemein unsichere Lage beseitigen könnte. Das Bild, das goldene Zeiten verheißt, bietet gleichzeitig die Auflösung “der unlösbaren Zweitteilung zwischen Natur und Zivilisation” (Popović, 2015, 257).

\section{Fazit}

Schlussfolgernd kann festgestellt werden, dass die typischen Darstellungen der unterschiedlichen räumlichen Aspekte in den Reiseberichten, die zur Zeit, als Njegoš regierte, entstanden sind, - trotz der Heterogenität der Erzählstrukturen in diesen Texten - ein mehr oder weniger einheitliches Bild Montenegros als besonderer oder im Vergleich zu westlichen Vorstellungen radikal anderer bzw. exotischer Raum vermitteln. Der Exotismus dieser Raumdarstellungen reicht vom überwiegend bedrohlichen Fremden über eine wilde, raue Andersartigkeit, die es zu zivilisieren gilt, bis hin zu der paradiesischen, gar utopischen Natürlichkeit, die bewahrt werden sollte. Dementsprechend wird dieser Exotismus sowohl positiv als auch negativ konnotiert. Er basiert vor allem auf der subjektiven Wahrnehmung des Reisenden, der sich in der Regel weder seiner Position als Subjekt des Narrativs noch der Subjektivität seiner Darstellungen klar bewusst ist. Und obwohl er zu einer "treuen Wiedergabe der Eindrücke" tendiert (Ebel, 1842-1844, III), basieren all seine Betrachtungen quasi (fast) unbewusst auf den ihm vertrauten räumlichen Strukturen der westlichen, 
europäischen Zivilisation, zu denen der montenegrinische Raum im Vergleich als anders erkannt wird. Die beschriebenen geographischen Strukturen werden anhand reicher Attribute, räumlicher Metaphorik und eines erweiterten Vergleichs sowie mittels bekannter räumlicher Symbole und auffälliger Kontrastierungen als exotische - einmal bedrohliche, einmal atemberaubende - Wildnis, die es noch zu zivilisieren gilt, semantisiert. Mit diesem Bild wird Montenegro als literarischer Schauplatz sowohl in weiteren Reiseberichten als auch in den fiktionalen Gattungen - bis tief in das 20. Jahrhundert assoziiert. Und wenn man heute an die EU-Integration und die Entwicklungen bei den Annäherungsbestrebungen Montenegros an Europa denkt, kann es sein, dass das Ganze auch außerliterarische Implikationen aufweist. Es erscheint durchaus vielversprechend, auch diesbezüglich noch interdisziplinäre Arbeiten dazu durchzuführen.

\section{Bibliographie}

\section{Quellen}

Ebel, W., Zwölf Tage auf Montenegro, H. 1-2, Königsberg 1842-1844.

Kohl, J. G., Reise nach Istrien, Dalmatien und Montenegro, Dresden 1851.

Müller, J., Albanien, Rumelien und die österreichisch-montenegrinische Gränze, Prag 1844.

Neigebaur, J. F., Die Süd-Slaven und deren Länder in Beziehung auf Geschichte, Cultur und Verfassung, Leipzig 1851.

Stieglitz, H., Ein Besuch auf Montenegro, Stuttgart, Tübingen 1841.

Stieglitz, H., Istrien und Dalmatien, Stuttgart, Tübingen 1845.

\section{Literatur}

Andrijašević, Ž., Istorija Crne Gore, Beograd 2016.

Bogojević, D., L'Imaginaire du Montenegro dans la littérature de voyage au XIX siècle et au debut du XX siècle, Paris 2011.

Bogojević, D., Viala de Somijer i Mario Serme o Crnoj Gori, Matica 7-8, II/2001, S. 217-247.

Badenberg, N., Exotismus, in: Metzler Lexikon Literatur (Hg. Burdorf, D. et al.), Stuttgart, Weimar 2007, S. 220-221.

Gazda, G., Tinecka Makovska, S., Rečnik književnih rodova i vrsta, Beograd 2016.

Karadžić, V. St., Montenegro und die Montenegriner, Ein Beitrag zur Kenntnis der europäischen Türkei und des serbischen Volkes, Stuttgart, Tübingen 1837. 
Konstantinović, Z., Deutsche Reisebeschreibungen über Serbien und Montenegro, München 1960.

Krause, F., Deutsche Reisende über Serbien und Montenegro um die Mitte des 19. Jahrhunderts, in: Serbien und Deutsche. Traditionen der Gemeinsamkeit gegen Feindbilder (Hg. Schubert, G. et al.), Jena, Erlangen 2003, S. 183-206.

Krivokapić, M., Diamond, N., Images of Montenegro in Anglo-American Creative Writing and Film, Cambridge 2017.

Lindau, W. A., Dalmatien und Montenegro. Mit einem Ausfluge nach der Herzegowina und einer geschichtlichen Uebersicht der Schicksale Dalmatiens und Ragusa's, Leipzig 1949.

Miljić, M., Prvi pomeni Crne Gore, Matica 67, 2016, S. 383-396.

Medenica, R., Nemci o Crnoj Gori i Crnogorcima, Cetinje i Crna Gora, Beograd 1927, S. 375-386.

Mojašević, M., Kulturno-posrednička uloga časopisa Das Ausland između Nemaca i Jugoslovena (1828-1893), in: Zbornik Filozofskog fakulteta III, Beograd 1955, S. 421-517.

Perišić, D., Deutsche Reisebeschreibungen über Montenegro im 19. Jahrhundert, Zeitschrift für Slavistik 26, 1981, S. 278-286.

Popović, O., Italijanski putopisi XIX vijeka o Crnoj Gori, 2015, unpub., S. 313.

Schuster, J., Reisebericht, in: Metzler Lexikon Literatur (Hg. Burdorf, D. et al.), Stuttgart, Weimar 2007, S. 640-641.

Petter, F., Skizze von Montenegro, in: Taschenbuch zur Verbreitung geographischer Kenntnisse, Prag 1832, S. 277-323.

Sommer, J. G., Zur Kenntnis von Montenegro, in: Taschenbuch zur Verbreitung geographischer Kenntnisse, Prag 1845, S. 235-275.

Wilkinson, J. G., Dalmatia and Montenegro, London 1848. 
Jelena Knežević, Ana Minić

\section{Montenegro als exotischer Raum in deutschsprachigen Reiseberichten in der ersten Hälfte des 19. Jahrhunderts}

Schlüsselwörter: Reiseberichte, Montenegro, Njegoš Zeiten, Raumdarstellung, exotisch, Exotismus

Der Aufsatz befasst sich mit der Perzeption des Raumes in deutschsprachigen Reiseberichten über Montenegro aus der ersten Hälfte des 19. Jahrhunderts. In sechs Reiseberichten, die in der Zeit, als Njegoš regierte (1830-1851), entstanden sind, wurden maßgebliche Aspekte der Darstellung des Raumes untersucht und identifiziert, die das Bild Montenegros als exotisches Land transportieren, unabhängig davon, ob der Exotismus als Form der Fokussierung auf das Fremde positiv oder negativ konnotiert ist. Es wurde festgestellt, dass der Exotismus der Darstellung des Raumes Montenegros in den interpretierten und analysierten Texten vor allem auf der Diskrepanz zwischen dem Bestreben einer objektiven Darstellung und der subjektiven Wahrnehmung des Erzählers basiert. Die beschriebenen geographischen Strukturen werden anhand unterschiedlicher literarischer Mittel als exotische, andere, merkwürdige, bedrohliche oder arkadische Wildnis semantisiert, womit Montenegro bis tief ins 20. Jahrhundert assoziiert wurde. 


\section{Jelena Knežević, Ana Minić \\ Črna gora kot eksotični prostor $v$ nemških potopisih iz prve polovice 19. stoletja}

Ključne besede: potopisi, Črna gora, Njegošev čas, upodobitve prostora, eksotično, eksotizem

Članek se ukvarja s percepcijo prostora v potopisih, napisanih v nemškem jeziku v prvi polovici 19. stoletja, ki opisujejo Črno goro. Raziskali sva šest potopisov, nastalih v času Njegoševe vladavine (1830-1851), glede na temeljne vidike upodobitve prostora in ugotovili, da posredujejo podobo Črne gore kot eksotične dežele, ne glede na to, ali je eksotizem kot oblika fokusiranja na tujost konotiran pozitivno ali negativno. Ugotovili sva, da eksotizem v upodobitvah črnogorskega prostora $\mathrm{v}$ analiziranih besedilih temelji predvsem na razkoraku med stremljenjem po objektivnosti poročanja na eni strani in pripovedovalčevim subjektivnim zaznavanjem na drugi. Besedila opisujejo geografske strukture ter jih z različnimi literarnimi sredstvi semantizirajo kot eksotično, drugačno, čudno in nevarno divjino ali kot Arkadijo. S tem tvorijo polje asociacij, ki se je s Črno goro povezovalo vse v 20. stoletje. 
Jelena Knežević, Ana Minić

\section{Montenegro as an Exotic Space in German Travelogues in the First Half of the $19^{\text {th }}$ Century}

Keywords: travelogues, Montenegro, Njegoš times, spatial representation, exotic, exoticism

This article deals with the perception of space in German travelogues about Montenegro written in the first half of the $19^{\text {th }}$ century. The significant aspects of the representation of space that transported the image of Montenegro as an exotic land were identified and examined in six travelogues that originated from the time of Njegoš rule (1830-1851). Exoticism as form of focusing on the foreign was partly positively and partly negatively connoted. It was found that the exoticism of the spatial representation of Montenegro in the interpreted and analysed texts is primarily based on the discrepancy between an intention to achieve an objective representation and the subjective perception of the narrator. By different literary means the described geographical structures were semantized as exotic, other, strange, and threatening or as the Arcadian wilderness, with which Montenegro was associated until late into the $20^{\text {th }}$ century. 